\title{
Five Key Ideas to Teach Fractions and Decimals with Understanding
}

\author{
Sam Strother \\ Boise State University \\ Jonathan L. Brendefur \\ Boise State University \\ Keith Thiede \\ Boise State University \\ Sarah Appleton, MAE \\ West Ada School District
}

\begin{abstract}
The teaching of fractions and decimals is a significant challenge for many teachers due to the inherent difficulty of the topic for students as well as the lack of high-quality, modernized curricular materials. This article examines the key ideas of teaching fractions and decimals for understanding that are evident in the current research literature and the curricular materials and teaching strategies from high-achieving nations.
\end{abstract}

Keywords: Fractions; Decimals; DMT

\section{INTRODUCTION}

The teaching and learning of fractions and decimals, with understanding, requires significant effort from teachers and students. These early rational number topics are often students' first experiences with levels of mathematical content that do not align well with students' intuitive notions of mathematics. For example, the very idea that as the denominator of a fraction increases the relative size of the unit fraction the denominator it is describing decreases is not a natural understanding for young learners. Similarly, it would seem that because decimal numbers are an extension of the base-10 whole numbers these new number should be described in ways that align directly with what students already know about whole numbers. However, as any teacher who has taught decimals to elementary students can attest, the concept of naming decimals by the smallest unit (e.g. .4 is said as "four tenths" but .40 is named "forty hundredths") is quite unusual for students who have spent most of their early years in school examining whole numbers. Unlike decimals, whole numbers are always named by the quantity of units of one that compose them. It is widely agreed that because of these unique and counter-intuitive aspects of fraction and decimal topics, students in upper-elementary grades and early middle-grades must be given significant time to learn the concepts and teachers need materials to implement effective instructional practices (NMAP 2008). Given that secondary level algebra classes require a substantial foundational understanding of fractions, decimals, and ratio and proportion concepts it is reasonable to assume that students leaving elementary school with this foundational knowledge are more likely to find success in their remaining school years. 
With the adoption of the Common Core Standards in many states, the need for high quality curricular materials that better embody the standards' learning progression for fractions and decimals are necessary. There has also been much research completed in the area of fractions and decimals within the last decade that could influence the teaching of them. Teachers will also be expected to use instructional strategies in the classroom that are effective in presenting fraction and decimal concepts to culturally and linguistically diverse learners as well as students with special needs.

At the [Center] we have been engaged in providing professional development, conducting research and supporting teachers in the classroom on the topic of fractions and decimals for several years. Based on our experiences and findings, we have determined there are five key ideas that help teachers promote student understanding and proficiency with fractions and decimals. Here we present the five ideas we have determined will help educators make informed decisions about new curricular materials that may be adopted in their school systems as well as the day to day practice of teaching fractions and decimals for understanding.

\section{EMPHASIZE THE CONCEPT OF UNITS}

Fractions and decimals, being a subset of the larger set of rational numbers, are structured on an essential concept of units. The term unit is often assumed to mean the whole number "one" but in the case of fractions the unit is actually defined by the denominator and can change as we count or measure with different fractions. In decimal numbers the unit is any decimal place value that is created by partitioning (equally splitting) the previous place value units into ten equivalent parts. This is much the same way all fractions are constructed but decimals are unique in that they follow our base-10 number system and are always partitions or iterations (copies) of ten of the previous smaller or larger unit.

Asking students to name fractions and decimals as collections of specific units can support their learning of unit fractions (any fraction with a numerator of 1 and a denominator that is a natural number, e.g. $\frac{1}{n}$ where $n$ is a whole number greater than 0 ). When students see the number $\frac{\mathbf{3}}{\mathbf{1 0}}$ or .3, they should initially name these numbers as, "three one-tenth units." To help students communicate their descriptions symbolically this description can be notated as $3\left(\frac{\mathbf{1}}{\mathbf{1 0}}-\right.$ units) or 3(.1-units) (Lamon 2012). The emphasis on unit fractions and units of decimal place value occurs most thoroughly in the Grade 3 Core Standards, but this emphasis on units is a key aspect of what students in later grades are expected to learn about equivalent fractions and operations with fractions and decimals (NGA 2010).

\section{Use Precise Definitions of the Numerator and Denominator}

A common definition in U.S. textbooks for the numerator and denominator is that the denominator is the parts to make a whole and the numerator is the number parts being counted (Lamon 2012). However, these definitions do not adequately represent the way fractions must be understood to extend students' learning beyond the simplest examples of these numbers nor are these definitions sufficient to apply to learning fraction and decimal operations (Wu 2011). Reviewing the history of these concepts reveals that the denominator is intended to identify, or name the unit being counted and therefore the size of this unit. For example, when examining the fraction $\frac{2}{3}$, the denominator of 3 tells us that each whole unit of 1 (not exclusively the unit length from 0 to 1 , but all whole unit lengths of 1 ) must be partitioned into three equivalent units. In a related way, the term numerator comes from the word "enumerate" 
which means to count. Therefore, in $\frac{2}{3}$ the denominator has defined the unit we are counting in by partitioning each whole unit of 1 into three equal parts and we have then counted, or "enumerated" two of these parts according to the numerator. As shown in Figures 1 and 2, when students learn the less accurate definitions of numerators and denominators as simply parts of a whole, confusion can occur when encountering fractions greater than one as well as when adding fractions using visual diagrams. Thinking of fractions as parts of a whole is very confusing if the numerator is greater than the denominator as students reasonably ask, "How can there be more parts than there are in the whole?" Students will often look at a model or context for an improper fraction such as $\frac{\mathbf{6}}{\mathbf{4}}$ and incorrectly name it as $\frac{\mathbf{6}}{\mathbf{8}}$. Likewise, adding fractions without clearly understanding the denominator indicates the size of the unit being counted and is always referencing the partitions of whole units of one can often lead early learners to incorrectly assume $\frac{\mathbf{1}}{\mathbf{3}}+\frac{\mathbf{1}}{\mathbf{3}}=\frac{\mathbf{2}}{\mathbf{6}}$. These potential confusions are lessened when students learn the more appropriate definitions we have described as there is no reason why counting $6\left(\frac{\mathbf{1}}{\mathbf{4}}\right.$ - units) is conceptually different than $3\left(\frac{\mathbf{1}}{\mathbf{4}}\right.$ - units) when the numerator is understood as a counting part of the fraction. Nor will students be compelled to add denominators when they understand that the denominator is the part of a fraction that determines the unit being counted and is always referring to the partitions of every whole unit of one, not the entire collection of units evident in the model or context.

Figure 1. Models of $\frac{3}{4}$ and $\frac{6}{4}$.

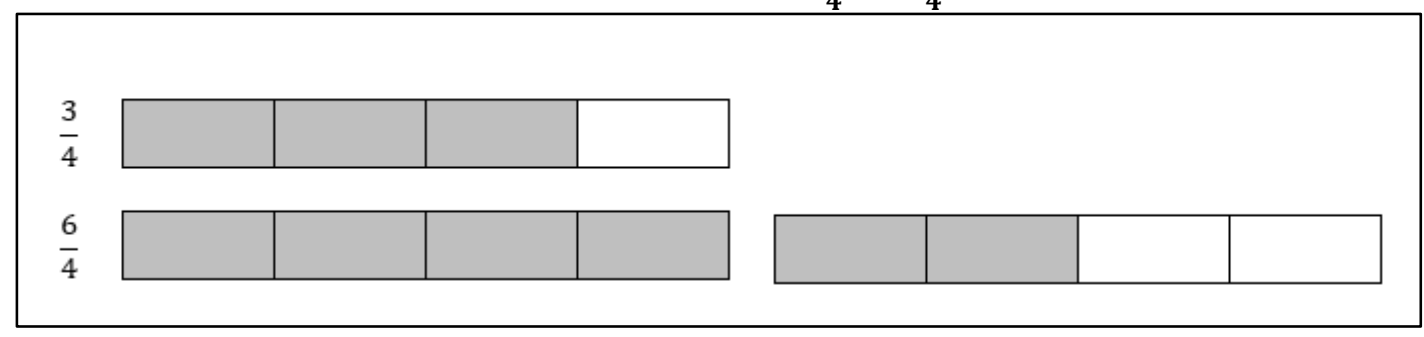

Figure 2. Example of Students' Common Fraction Addition Misconception: $\frac{1}{3}+\frac{1}{3}=\frac{2}{6}$.

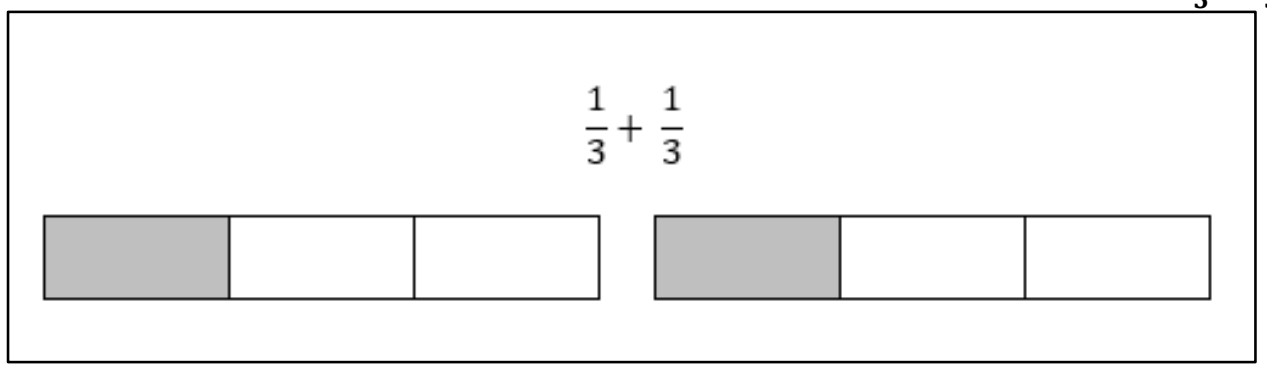

Introduce bar models and number lines as initial representations of fraction and decimals To examine the sophisticated concepts associated with fractions \& decimals, a wellestablished and time honored instructional technique is to use visual models and diagrams to support students' learning (Lamon 2012). Unfortunately, there are many potential confusions that certain fraction models may create for early learners (Watanabe 2002). Specifically, models such as set models (e.g. collections of discrete objects) and region models (e.g. rectangular areas \& circles) shown in Fig. 3 should be utilized as instructional tools later in the students' learning process because of their tendency to convolute the intended knowledge and skills to be acquired. In the case of set models, examining a collection objects can lead students to believe that fractions with greater denominators are larger as the set used to create these fractions is indeed greater than the sets to model fractions with smaller denominators. When 
describing set models as fractions, the fraction name is actually describing a conceptually abstract ratio of part to whole that is not directly observable to many students.

When asking students to use region models to learn fraction and decimal concepts, there is ample risk of confusion as two-dimensional space is often difficult to compare directly. A rectangle or circle may be partitioned into two equal parts in multiple ways, but as shown in Figure 3, the portions of area meant to be unit fractions are often very different in shape and therefore difficult to compare without further understanding of area formulas.

When bar models and number lines are used to compare fractions or model fraction concepts, the unit lengths are more easily compared than two-dimensional regions and the whole unit of one is more easily established than when using set models. Therefore, early fraction and decimal learning should likely afford students more time to become proficient with creating, describing and examining bar models and number lines. This is an approach that is common in many high-achieving nations' curricular materials for fractions and decimals (Alajmi 2012, Leinwand and Ginsburg 2007, Watanabe 2002).

Figure 3. Models Used to Compare $\frac{1}{5}$ and $\frac{1}{4}$.

Bar Models
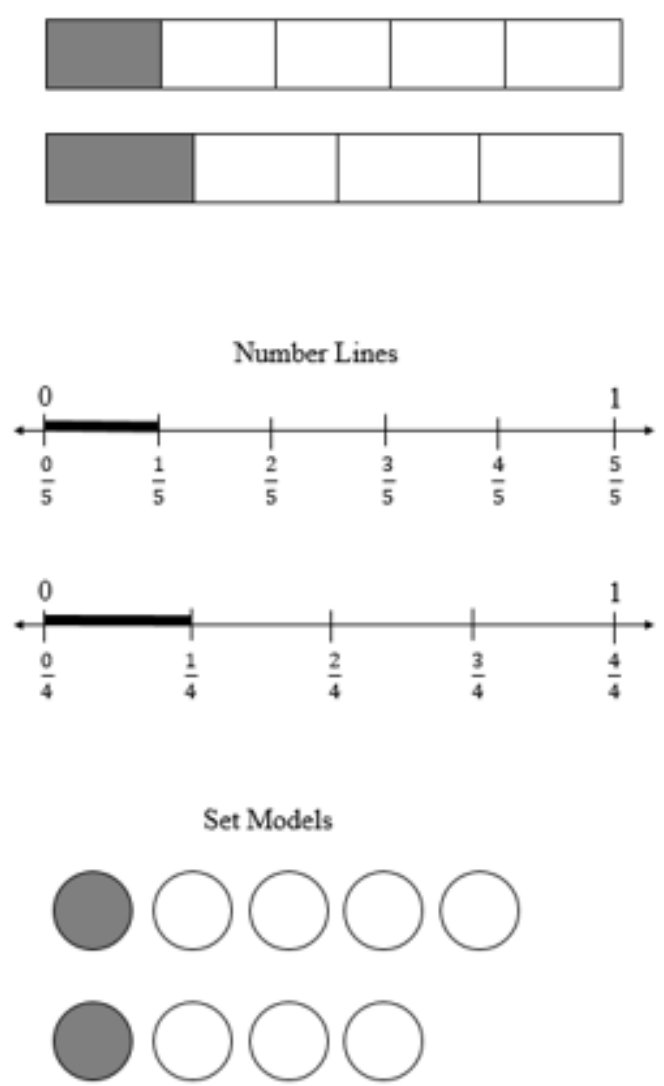

Region Models: Rectangular Areas
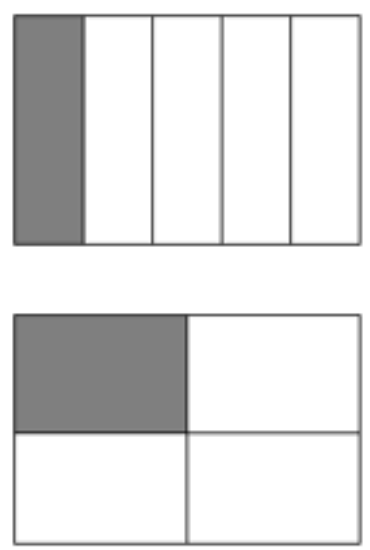

Region Models: Circle Fractions
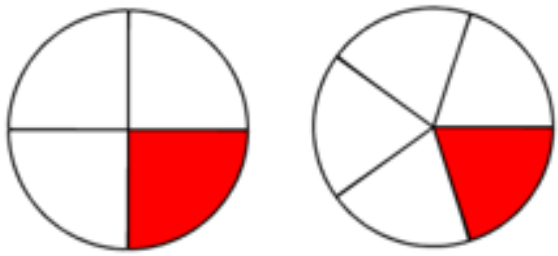

Assign tasks that require students to reason about the correctness of another student's thinking

An under-utilized but extremely informative assessment technique found in many highly regarded assessment instruments is to present students with another student's reasoning on a non-routine problem. This fictional student's reasoning may often contain flaws and 
misconceptions that arise from deep conceptual misunderstandings (de Lange 1999, Webb 2002). By asking students to first determine why a fictional peer might reasonably determine $\frac{1}{3}+\frac{1}{3}=\frac{2}{6}$ or that $.4+.8=.12$, teachers create an opportunity to alleviate potential misconceptions and misunderstandings before they become solidified in students' thought processes. This strategy also allows learners to more clearly articulate the error in understanding and then provide a justification to support the fictional student in correcting the error, thus building a foundation for self-reflection and individual error-analysis approaches when new information is encountered. There is also a view that this approach is part of what it means to engage in the practice of mathematical thinking. The Core Standards include this approach as one of the eight mathematical practices, “...construct viable arguments and critique the reasoning of others." (NGA 2010).

\section{Support Student Discussion With Structured Language Guides}

Students learn a great deal by discussing their own ideas with a partner, small group, or entire class (Kazemi 1998). However, given the sophistication of fraction and decimal content it is probably unwise to presume students will have the necessary academic vocabulary and technical language skills to engage in meaningful discussions about the content. Therefore, providing students with linguistic supports to help guide their discourse is a highly effective technique with ample supporting evidence from the field of English language development (Gersten et al. 2007). By including word walls (posters of key academic vocabulary terms) as part of the classroom environment and by providing students with examples of high-quality mathematical discussions in the form of sentence starters (brief, partial phrases that model how to begin expressing an idea or ask meaningful questions) teachers structure their students' peer-to-peer conversations. In this way, students from diverse linguistic backgrounds as well as fluent English speakers are given the necessary assistance in communicating their ideas as they learn very difficult fraction and decimal content (Gersten et al. 2007).

\section{Do These Five Ideas Change How Well Students Understand Fractions And Decimals?}

To establish these five key ideas actually do have an effect on student learning of fractions and decimals, the [Center] developed a module consisting of 10 lessons and a pre/post-test that included many of the important fraction concepts found in the $3^{\text {rd }}-4^{\text {th }}$ grade Core Standards and built around the elements of developing mathematical thinking (Brendefur et al, 2013). The entire module, including the pre and post-tests took approximately 3 weeks to complete. The module was built with these five key ideas as the foundation and was then provided to 3$4^{\text {th }}$ grade teachers. Other teachers in grades 3 and 4 gave the pre and post-tests for comparative purposes and taught fractions during the same time period using their already available curricular materials, which based on a review from [Center] staff, did not adequately embody the five key ideas. The results shown in Table 1 indicate that students learning fractions from the module constructed with these five key elements significantly outperformed students who learned fractions and decimals during the same time period from materials that did not exemplify these five ideas.

Table 1. Mean Test Performance by Group across Time

\begin{tabular}{lll}
\hline Group & Pretest & Pottest \\
\hline Modules & $3.68(.39)$ & $8.83(.39)$ \\
No Modules & $5.69(.39)$ & $7.91(.39)$ \\
\hline
\end{tabular}

Note. The numbers in parentheses are the standard error of the mean. 


\section{CONCLUSION}

It is our intent to provide these five recommendations as guidance for teachers and administrators who will be charged with examining new curricular materials and then implementing instructional strategies to fulfill the intended learning of these materials. If new materials aligned to the Core Standards do indeed contain these five key ideas it is likely they will afford teachers an opportunity to engage in effective instructional practices when teaching fractions and decimals. In the meantime, these five key ideas can serve as a guide for modifying or supplementing currently available curricular materials so that students can learn fractions and decimals with understanding.

\section{References}

Alajmi, Amal Hussain. 2012. "How do elementary textbooks address fractions? A review of mathematics textbooks in the USA, Japan, and Kuwait." Educational Studies in Mathematics 79 (2):239-261.

Brendefur, J. L., Thiede, K., Strother, S., Bunning, K., \& Peck, D. (2013). Developing mathematical thinking: Changing teachers' knowledge and instruction. Journal of Curriculum and Teaching, 2(2).

de Lange, Jan. 1999. Framework for classroom assessment in mathematics. Unpublished Manuscript. Madison, WI.

Gersten, R., S. K. Baker, P. Collins, S. Linan-Thompson, and T. Shanahan. 2007. Effective literacy and English language instruction for English learners in the elementary grades. Washington, D.C.: Institute of Education Sciences.

Kazemi, Elham. 1998. "Discourse that promotes conceptual understanding." Teaching Children Mathematics 4 (7):410-414.

Lamon, Susan J. 2012. Teaching fractions and ratios for understanding: Essential content knowledge and instructional strategies for teachers: Routledge.

Leinwand, Steven, and Alan L Ginsburg. 2007. "Learning from Singapore math." Educational Leadership 65 (3):3236.

NGA. 2010. Common core state standards for mathematics. Washington DC: National Governors Association and the Council of Chief State School Officers.

NMAP. 2008. Foundations for success: The final report of the National Mathematics Advisory Panel. Edited by National Mathematics Advisory Panel. Washington D.C.: U.S. Department of Education, Office of Planning, Evaluation and Policy Development.

Watanabe, T. 2002. "Representations in teaching and learning fractions." Teaching Children Mathematics 8 (8):457-563.

Webb, Norman L. 2002. "Depth-of-knowledge levels for four content areas." Language Arts.

Wu, H. 2011. "The mis-education of mathematics teachers." Notices of the AMS 58 (3):372-384. 